\title{
On the spinor representation
}

\author{
J. M. Hoff da Silva ${ }^{1, a}$, C. H. Coronado Villalobos ${ }^{1,2, b}$, Roldão da Rocha ${ }^{3, c}$, R. J. Bueno Rogerio ${ }^{1, d}$ \\ ${ }^{1}$ Departamento de Física e Química, Universidade Estadual Paulista, Guaratinguetá, SP, Brazil \\ ${ }^{2}$ Instituto de Física, Universidade Federal Fluminense, Av. Gal. Milton Tavares de Souza s/n, Gragoatá, Niterói, RJ 24210-346, Brazil \\ ${ }^{3}$ Centro de Matemática, Computação e Cognição, Universidade Federal do ABC-UFABC, Santo André 09210-580, Brazil
}

Received: 16 February 2017 / Accepted: 30 June 2017 / Published online: 21 July 2017

(C) The Author(s) 2017. This article is an open access publication

\begin{abstract}
A systematic study of the spinor representation by means of the fermionic physical space is accomplished and implemented. The spinor representation space is shown to be constrained by the Fierz-Pauli-Kofink identities among the spinor bilinear covariants. A robust geometric and topological structure can be manifested from the spinor space, wherein the first and second homotopy groups play prominent roles on the underlying physical properties, associated to fermionic fields. The mapping that changes spinor fields classes is then exemplified, in an Einstein-Dirac system that provides the spacetime generated by a fermion.
\end{abstract}

\section{Introduction}

The very definition of a spinor in dealing with physics may be treated as a matter of some importance in itself whatsoever. In fact, from simple quaternionic compositions revealing a definite rotation [1] to the fermionic quantum internal structure [2], the spinorial approach reveals its richness. Among these possible systematizations concerning spinors, there is a particularly relevant one that encodes all the algebraically necessary information and the important relativistic construction as well, namely, the multivector spinor representation. When represented as a section of a bundle comprised by the $S L(2, \mathbb{C})$ group and $\mathbb{C}^{4}$, it is possible to understand several spinor properties by inspecting the multivector part constructed out specific $S L(2, \mathbb{C})$ objects. These objects are nothing but the bilinear covariants associated to the regarded spinor [3,4].

Following this reasoning, the usefulness of such a representation is not surprising, since the bilinear covariants are,

\footnotetext{
a e-mail: hoff@feg.unesp.br

be-mails: ccoronado@feg.unesp.br; ccoronado@id.uff.br

c e-mail: roldao.rocha@ufabc.edu.br

de-mail: rodolforogerio@feg.unesp.br
}

at least in principle, related to a set of fermionic observables. Our aim in this paper is to delineate the importance of the representation space, by studying its properties, and then relating them to their physical consequences. As one will realize, the representation space is quite complicated due to the constraints coming out the Fierz-Pauli-Kofink identities. However, a systematic study of the space properties ends up being useful to relate different domains (subspaces) to the corresponding physics. Moreover, this study permits the study of fermions from a different and useful perspective.

This paper is organized as follows: in the next section the standard framework and the three equivalent definitions of spinors are revisited for the Minkowski spacetime, emphasizing the most relevant aspects concerning our purposes. Section 3 is devoted to our approach to the Lounesto spinor classification and related issues. In Sect. 4 we construct and study the spinor representation space and explore the topological and physical consequences. Section 5 is devoted to a derivation of the spacetime around a self-interacting spinor field that satisfies the Dirac equation coupled to the Einstein equations with cosmological constant. We show that, for periodic values of the time variable, regular spinors are led into flag-dipoles, dynamically implementing the algebraic mapping proposed in Sect. 6. In the final section we conclude.

\section{The three equivalent definitions of spinors}

Consider the Minkowski spacetime $\left(M \simeq \mathbb{R}^{4}, \eta_{\mu \nu}\right)$ and its tangent bundle $T M$, where $\eta$ denotes the Minkowski metric and Greek (spacetime) indices run from 0 to 3. Denoting sections of the exterior bundle by $\sec \Omega(M)$, the spacetime Clifford algebra shall be denoted by $\mathcal{C} \ell_{1,3}$. The set $\left\{e_{\mu}\right\}$ represents sections of the frame bundle $\mathbf{P}_{\mathrm{SO}_{1,3}^{e}}(M)$, whereas the set $\left\{\gamma^{\mu}\right\}$ can be further thought of as being the dual basis, $\gamma^{\mu}\left(e_{v}\right)=\delta_{v}^{\mu}$. Classical spinors are objects of the space that carry the usual $\tau=(1 / 2,0) \oplus(0,1 / 2)$ representations of 
the Lorentz group, which can be thought of as being sections of the vector bundle $\mathbf{P}_{\operatorname{Spin}_{1,3}^{e}}(M) \times_{\tau} \mathbb{C}^{4}[5,6]$.

The underlying idea that can join the three definitions of spinors relies on a quite straightforward root, and it was inspired by the spacetime algebra, whose elements satisfy $e_{\mu} e_{\nu}+e_{\nu} e_{\mu}=2 \eta_{\mu \nu} 1$. Indeed, any arbitrary element $\Pi=s+s^{\mu} e_{\mu}+s^{\mu \nu} e_{\mu} e_{\nu}+s^{\mu \nu \tau} e_{\mu} e_{\nu} e_{\tau}+p e_{0} e_{1} e_{2} e_{3} \in$ $\mathcal{C} \ell_{1,3}$ has a quaternionic representation. By denoting $\mathbb{H}$ the quaternionic ring, a spinor representation of the Clifford algebra $\mathcal{C} \ell_{1,3} \simeq \mathcal{M}(2, \mathbb{H})$ can be derived. A primitive idempotent $f=\frac{1}{2}\left(1+e_{0}\right)$ defines a minimal left ideal $\mathcal{C} \ell_{1,3} f$, whose arbitrary element can be expressed as $[7,8]$

$$
\begin{aligned}
\xi= & {\left[\left(s+s^{0}\right)+\left(s^{23}+s^{023}\right) e_{2} e_{3}\right.} \\
& \left.-\left(s^{13}+s^{013}\right) e_{3} e_{1}+\left(s^{12}+s^{012}\right) e_{1} e_{2}\right] f \\
& +\left[\left(p-s^{123}\right)+\left(s^{1}-s^{01}\right) e_{2} e_{3}\right. \\
& \left.+\left(s^{2}-s^{02}\right) e_{3} e_{1}+\left(s^{3}-s^{03}\right)\right] e_{0} e_{1} e_{2} e_{3} f,
\end{aligned}
$$

constituting an algebraic spinor $\xi \in \mathcal{C} \ell_{1,3} f$. The set comprised by the units $i=e_{2} e_{3}, j=e_{3} e_{1}$, and $\hat{k}=e_{1} e_{2}$ settles a basis for the quaternionic algebra $\mathbb{H}$.

Representations of the $\left\{e_{\mu}\right\}$ in $\mathcal{M}(2, \mathbb{H})$ read [9]

$$
\begin{aligned}
& {\left[e_{1}\right]=\left(\begin{array}{ll}
0 & i \\
i & 0
\end{array}\right), \quad\left[e_{2}\right]=\left(\begin{array}{ll}
0 & j \\
j & 0
\end{array}\right),} \\
& {\left[e_{3}\right]=\left(\begin{array}{ll}
0 & \mathfrak{k} \\
\mathfrak{k} & 0
\end{array}\right), \quad\left[e_{0}\right]=\left(\begin{array}{cc}
1 & 0 \\
0 & -1
\end{array}\right) .}
\end{aligned}
$$

Then the elements $f$ and $e_{0} e_{1} e_{2} e_{3} f$ have, respectively, the representations $[f]=\left(\begin{array}{ll}1 & 0 \\ 0 & 0\end{array}\right)$ and $\left[e_{0} e_{1} e_{2} e_{3} f\right]=\left(\begin{array}{ll}0 & 0 \\ 1 & 0\end{array}\right)$ [10]. Hence, an arbitrary element $\Psi \in \mathcal{C} \ell_{1,3}^{+}$, in the even subalgebra, corresponds to the so-called spinor operator [3]

$\Psi=s+s^{\mu v} e_{\mu} e_{v}+p e_{0} e_{1} e_{2} e_{3} \simeq\left(\begin{array}{cc}q_{1} & -q_{2} \\ q_{2} & q_{1}\end{array}\right) \in \mathcal{M}(2, \mathbb{H})$,

where, according to Eq. (1), we have

$q_{1}=s+s^{23} \mathfrak{i}+s^{31} \mathfrak{j}+s^{12} \mathfrak{k}$,

$q_{2}=-p+s^{01} \mathbf{i}+s^{02} \mathbf{j}+s^{03} \hat{k}$.

The vector space isomorphisms $\mathbb{H}^{2} \simeq \mathcal{C} \ell_{1,3}^{+} \simeq \mathbb{C}^{4} \simeq \mathcal{C} \ell_{1,3} f$ constitute the landmark for the relationship among the spinor operator, the algebraic, and the classical definitions of a spinor $[6,11]$. Hence, it is possible to alternatively write the Dirac algebraic spinor field as an element $\left(\begin{array}{l}q_{1} \\ q_{2}\end{array}\right)$ of the ring $\mathbb{H} \oplus \mathbb{H}$, as [7-9]

$$
\left(\begin{array}{cc}
q_{1} & -q_{2} \\
q_{2} & q_{1}
\end{array}\right)[f] \in \mathcal{C} \ell_{1,3} f
$$

Returning to Eq. (3), and using for instance the standard representation, the complex matrix associated to the spinor operator $\Psi$ in (3) reads

$$
\begin{aligned}
{[\Psi] } & =\left(\begin{array}{cc}
\mathbb{A} & -\mathbb{B} \\
\mathbb{B} & \mathbb{A}
\end{array}\right), \quad \text { for } \mathbb{A}=\left(\begin{array}{cr}
\psi_{1} & -\psi_{2}^{*} \\
\psi_{2} & \psi_{1}^{*}
\end{array}\right), \\
\mathbb{B} & =\left(\begin{array}{ll}
\psi_{3} & -\psi_{4}^{*} \\
\psi_{4} & \psi_{3}^{*}
\end{array}\right),
\end{aligned}
$$

where

$$
\begin{aligned}
& \psi_{1}=s+s^{23} i, \quad \psi_{2}=s^{13}+s^{12} i \\
& \psi_{3}=p+s^{10} i, \quad \psi_{4}=s^{02}+s^{30} i .
\end{aligned}
$$

The standard Dirac spinor $\psi$ was identified, e.g., in Ref. [3] as an element of the minimal left ideal $\left(\mathbb{C} \otimes \mathcal{C} \ell_{1,3}\right) f$ associated to the complexified spacetime algebra $\left(\mathbb{C} \otimes \mathcal{C} \ell_{1,3}\right)$, generated by the primitive idempotent [3]

$f=\frac{1}{4}\left(1+e_{0}\right)\left(1+i e_{1} e_{2}\right)=\left(\begin{array}{cccc}1 & 0 & 0 & 0 \\ 0 & 0 & 0 & 0 \\ 0 & 0 & 0 & 0 \\ 0 & 0 & 0 & 0\end{array}\right)$,

yielding $\psi=\Pi \frac{1}{2}\left(1+i \gamma_{1} \gamma_{2}\right) \in\left(\mathbb{C} \otimes \mathcal{C} \ell_{1,3}\right) f$, with the identification $e_{\mu} \mapsto \gamma_{\mu}$. It yields the bijection between the algebraic spinor $[5,6,12]$

$\psi=\left(\begin{array}{llll}\psi_{1} & 0 & 0 & 0 \\ \psi_{2} & 0 & 0 & 0 \\ \psi_{3} & 0 & 0 & 0 \\ \psi_{4} & 0 & 0 & 0\end{array}\right) \in\left(\mathbb{C} \otimes \mathcal{C} \ell_{1,3}\right) f \simeq \mathcal{M}(4, \mathbb{C})$

and the classical one $\psi=\left(\psi_{1}, \psi_{2}, \psi_{3}, \psi_{4}\right)^{\top} \in \mathbb{C}^{4}$.

Given a representation $\rho: \mathbb{C} \otimes \mathcal{C} \ell_{1,3} \rightarrow \mathcal{M}(4, \mathbb{C})$, the adjoint of $A \in \mathbb{C} \otimes \mathcal{C} \ell_{1,3}$, defined by $A^{\dagger}=\rho^{-1}\left(\rho(A)^{\dagger}\right)$ (where $\rho(A)^{\dagger}$ denotes the standard Hermitian conjugation in $\mathcal{M}(4, \mathbb{C})$ ), reads $A^{\dagger}=e_{0} \tilde{A}^{*} e_{0}$, where $\tilde{A}$ stands for the reversion of $A$ and $(\cdot)^{*}$ denotes the complex conjugation. Besides, its trace is given by $\operatorname{Tr}(\rho(\psi))=4\langle\psi\rangle_{0}$, where this notation is used to indicate the projection of a multivector onto its scalar part.

This correspondence provides an immediate identification between $\psi$ and the classical Dirac spinor field. Having recovered the equivalence between these current spinor definitions, we pass to the building blocks of the spinorial representation space, namely the Fierz aggregate and the bilinear identities, after which we define the space itself, allowing for the connection of its points to a physical spinor, regardless the chosen classical definition. 


\section{Lounesto's spinor classification, Pauli-Fierz-Kofink identities, and the Fierz aggregate}

Any spinor field $\psi \in \sec \mathbf{P}_{\operatorname{Spin}_{1,3}^{e}}(M) \times{ }_{\tau} \mathbb{C}^{4}$ can be employed to construct its bilinear covariants as section of bundle $\Omega(M)$, reading $[3,4,13]$

$$
\begin{aligned}
\sigma & =\bar{\psi} \psi \in \Omega^{0}(M), \\
\omega & =-\bar{\psi} \gamma_{0} \gamma_{1} \gamma_{2} \gamma_{3} \psi \in \Omega^{4}(M), \\
\mathbf{J} & =\left(\bar{\psi} \gamma_{\mu} \psi\right) \gamma^{\mu} \in \Omega^{1}(M), \\
\mathbf{K} & =i\left(\bar{\psi} \gamma_{0} \gamma_{1} \gamma_{2} \gamma_{3} \gamma_{\mu} \psi\right) \gamma^{\mu} \in \Omega^{1}(M), \\
\mathbf{S} & =\left(\bar{\psi}\left[\gamma_{\mu}, \gamma_{\nu}\right] \psi\right) \gamma^{\mu} \wedge \gamma^{\nu} \in \Omega^{2}(M) .
\end{aligned}
$$

Equivalently, the components of the bilinear covariants are, respectively, denoted by

$$
\begin{aligned}
J_{\mu} & =\bar{\psi} \gamma_{\mu} \psi, \\
K_{\mu} & =i \bar{\psi} \gamma_{0} \gamma_{1} \gamma_{2} \gamma_{3} \gamma_{\mu} \psi, \\
S_{\mu \nu} & =\bar{\psi}\left[\gamma_{\mu}, \gamma_{\nu}\right] \psi .
\end{aligned}
$$

The bilinear covariants in the Dirac theory are interpreted respectively as the mass term (or invariant length) in the Lagrangian of the electron $(\sigma)$, the pseudo-scalar $(\omega)$ relevant for parity-coupling, the current of probability density $(\mathbf{J})$, the chiral current density $(\mathbf{K})$, and the probability density of the (intrinsic) electromagnetic moment $(\mathbf{S})[3,4]$. A prominent requirement for the Lounesto spinor classification is that the bilinear covariants satisfy quadratic algebraic relations, namely, the so-called Fierz-Pauli-Kofink (FPK) identities, which read

$$
\begin{aligned}
\mathbf{J}^{2} & \equiv J^{\mu} J_{\mu}=\omega^{2}+\sigma^{2}, \\
\mathbf{K}^{2} & \equiv K^{\mu} K_{\mu}=-\mathbf{J}^{2}, \\
\mathbf{J} \cdot \mathbf{K} & \equiv J^{\mu} K_{\mu}=0, \\
\mathbf{J} \wedge \mathbf{K} & =-\left(\omega+\sigma \gamma_{0} \gamma_{1} \gamma_{2} \gamma_{3}\right) \mathbf{S} .
\end{aligned}
$$

When an arbitrary spinor $\xi$ satisfies $\gamma_{0} \xi^{\dagger} \gamma_{0} \psi \neq 0$, the original spinor $\psi \neq 0$ can be reconstructed, using the aggregate

$\mathbf{Z}=\sigma+\mathbf{J}+i \mathbf{S}+i \mathbf{K} \gamma_{0} \gamma_{1} \gamma_{2} \gamma_{3}+\omega \gamma_{0} \gamma_{1} \gamma_{2} \gamma_{3}$

by (a version) of the inversion theorem, $\psi=\frac{1}{2 \sqrt{\xi^{\dagger} \gamma_{0} \mathbf{Z} \xi}} e^{-i \theta}$ $\mathbf{Z} \xi$, where $\theta=i \log \left(2\left(\xi^{\dagger} \gamma_{0} \psi \xi^{\dagger} \gamma_{0} \mathbf{Z} \xi\right)^{-1 / 2}\right)$ is $[4,14$, 15]. Moreover, when $\sigma, \omega, \mathbf{J}, \mathbf{S}, \mathbf{K}$ satisfy the Fierz identities, then the complex multivector operator $\mathbf{Z}$ is called a Fierz aggregate, which can be self-adjoint, being called a "boomerang" in this case [3]. The regular spinors are those whose at least one of the bilinear covariants $\sigma$ and $\omega$ do not vanish. On the other hand, singular spinors present $\sigma=0=\omega$, and, in this case, the Fierz identities, given in Eq. (13a), are in general replaced by the most general conditions [4]:

$$
\frac{1}{4} \mathbf{Z}^{2}=\sigma \mathbf{Z}, \quad \frac{1}{4} \mathbf{Z}_{\mu} \mathbf{Z}=J_{\mu} \mathbf{Z}, \quad \frac{1}{4} \mathbf{Z} i\left[\gamma_{\mu}, \gamma_{\nu}\right] \mathbf{Z}=S_{\mu \nu} \mathbf{Z},
$$

$\frac{1}{4} \mathbf{Z}_{i} \gamma_{0} \gamma_{1} \gamma_{2} \gamma_{3} \gamma_{\mu} \mathbf{Z}=K_{\mu} \mathbf{Z}, \quad-\frac{1}{4} \mathbf{Z} \gamma_{0} \gamma_{1} \gamma_{2} \gamma_{3} \mathbf{Z}=\omega \mathbf{Z}$.

These conditions are satisfied also by regular spinors. Such relations are called more general Fierz-Pauli-Kofink identities, however, they are written based on the Fierz aggregate.

Moreover, the inversion theorem (to be further studied in the next section) is inspired by this spinor representation. More significantly here, the aggregate plays a central role within the Lounesto classification since, in order to complete the classification itself, $\mathbf{Z}$ has to be promoted to a boomerang, satisfying $\mathbf{Z}^{2}=4 \sigma \mathbf{Z}$. Obviously, for regular spinors the above condition is satisfied and $\mathbf{Z}$ is automatically a boomerang. However, for the singular spinor case it is not so direct. Indeed, for singular spinors we must envisage the underlying geometric structure to the multivector. From the geometric point of view, the following relations between the bilinear covariants must be fulfilled, in order to ensure that the aggregate is a boomerang: $\mathbf{J}$ field must be parallel to $\mathbf{K}$ and both are elements in the plane formed by the bivector field $\mathbf{S}$. Hence, using the Eq. (14) and taking into account singular spinors, it is straightforward to see that the aggregate can be recast as [3]

$\mathbf{Z}=\mathbf{J}\left(1+i \mathbf{s}+i h \gamma_{0} \gamma_{1} \gamma_{2} \gamma_{3}\right)$,

where $\mathbf{s}$ is a space-like vector orthogonal to $\mathbf{J}$, and $h$ is a real scalar that is related to the spinor helicity. The multivector as expressed in Eq. (16) is a boomerang [16]. The condition $\mathbf{Z}^{2}=4 \sigma \mathbf{Z}$ yields $\mathbf{Z}^{2}=0$, for singular spinors have the property that, namely, the Fierz aggregate is nilpotent. However, for the FPK identities to hold, the vector field $J$ must be light-like (isotropic) and the multivector $h \gamma_{0} \gamma_{1} \gamma_{2} \gamma_{3}+\mathbf{s}$ must be a pure imaginary $[3,16]$.

With these ingredients, it is possible to envisage six different classes of spinors, according to the classification in Table 1.

The three first classes are composed of regular spinors that comprise the standard textbook Dirac spinor. As stated in the

Table 1 Lounesto's spinor field classification

\begin{tabular}{llllll}
\hline Class & $\sigma$ & $\omega$ & $\mathbf{K}$ & $\mathbf{S}$ & $\mathbf{J}$ \\
\hline 1 & $\neq 0$ & $\neq 0$ & $\neq 0$ & $\neq 0$ & $\neq 0$ \\
2 & $\neq 0$ & 0 & $\neq 0$ & $\neq 0$ & $\neq 0$ \\
3 & 0 & $\neq 0$ & $\neq 0$ & $\neq 0$ & $\neq 0$ \\
4 & 0 & 0 & $\neq 0$ & $\neq 0$ & $\neq 0$ \\
5 & 0 & 0 & 0 & $\neq 0$ & $\neq 0$ \\
6 & 0 & 0 & $\neq 0$ & 0 & $\neq 0$ \\
\hline
\end{tabular}


literature, the representation spaces for the mentioned spinors are linked by the parity symmetry, however, quite recently regular spinors have been shown to be built without reference to this symmetry [17]. The elements of the fifth class are also called flag-pole spinors, represented by particular cases as Majorana and Elko spinors, whereas the sixth class comprises Weyl spinors. The fourth class, the flag-dipole, has had its first physical example discovered recently [18]. For later reference we stress that $\mathbf{J}$ is always non-null within this context. The Lounesto classification has been explored in a comprehensive range of contexts, comprising field theory [22,23], cosmology [24], gravitation [25] and formal aspects as well $[16,26,27]$. The general form of the spinors in each one of the above classes was derived in Refs. $[18,28]$, and a classification that encodes gauge aspects was established in Ref. [29].

\section{The representation space}

Bearing in mind that a given spinor can be written as a section of the bundle $\mathbf{P}_{\operatorname{Spin}_{13}^{e}}(M) \times{ }_{\tau} \mathbb{C}^{4}$ we shall envisage the spinor space structure adopting a bottom-up, and somewhat pragmatic, approach by defining the regarded manifolds and spaces with respect to their points and elements. Notice that, as emphasized throughout Sect. 2, the understanding of spinors as sections of the aforementioned bundle are not strictly necessary, although highly convenient as we shall see.

In what follows let us denote by $\stackrel{\circ}{N}$ the 5-dimensional manifold whose points are in $\sec \Omega^{a}(M)$, with $a=0, \ldots, 4$. The space $\stackrel{\circ}{N}$ is isomorphic to the exterior bundle $\Omega(M)=$ $\oplus_{a=0}^{4} \Omega(M)$. Let us denote by $P=\left(p^{0}, p^{1}, p^{2}, p^{3}, p^{4}\right)$ an arbitrary point of the manifold $\stackrel{\circ}{N}$, and the function $Z$ that establishes such a canonical isomorphism $\stackrel{\circ}{\simeq} \Omega(M)$. Obviously $Z(P) \in \Omega(M)$.

Definition $1 \stackrel{\circ}{\Sigma}$ is the space whose elements are given by $Z \eta$, where $\eta \in \mathbb{C}^{4}$.

Notice that as long as $Z$ is restricted to the bilinear covariants, namely, we impose the requirement that it acts only upon points of $N$ satisfying the FPK identities, then the Fierz aggregate is straightforwardly obtained. Equivalently, however, more generally, we proceed with the following direct construction.

Definition $2 N$ is a submanifold of $\stackrel{\circ}{N}$ whose points are such that $Z(P)$ obeys the FPK identities.

When acting upon elements of $M$ it is convenient to write $Z(P)$ as

$Z(P)=\sigma+\mathbf{J}+\mathbf{K}+\mathbf{S}+\omega$,

making explicit the multivector structure in terms of the bilinear covariants, just as to express $P=(\sigma, \mathbf{J}, \mathbf{K}, \mathbf{S}, \omega)$.
Definition 3 The representation space $\Sigma(N)$ is performed by elements given by $Z(N) \eta$, where $Z(N)$ stands for $Z(P)$ with $P \in N$ only. Therefore $Z \eta \cong \Psi \in \Sigma(N)$ and the elements of $\Sigma$ are, thus, physical spinors.

It is worth emphasizing that since the bilinears are invariant with respect to Lorentz transformation, the elements of $\Sigma(N)$ are relativistically covariant. Clearly $\Sigma(N) \subset \stackrel{\circ}{\Sigma}$, i.e., the representation space is contained in the broader spinorial space. Therefore, the complement space $\stackrel{\circ}{\Sigma} \backslash \Sigma(N)$ comprises points corresponding to spinors which do not obey the FPK identities, the so-called anomalous spinors.

The underlying idea to this construction regards the possibility to change from one physical spinor configuration to another one, by covering a given continuous path in the representation space. Differently from what happens to $\stackrel{\circ}{N}$, however, the submanifold $N$ must have a quite constrained topology inherited from the validity of the FPK identities.

Let us make this point clearer by considering merely regular spinors for a moment. In this case, it is possible to attain the appropriate subspace of $\Sigma(N)$ by defining the following canonical projector $\xi_{\text {reg }}$ :

$$
\xi_{\text {reg }}: N \rightarrow \Omega(M)
$$

$(\sigma, \mathbf{J}, \mathbf{K}, \mathbf{S}, \omega) \mapsto(\sigma, \mathbf{J}, 0,0, \omega)$,

with image $\Omega^{0}(M) \oplus \Omega^{1}(M) \oplus \Omega^{4}(M)=\xi_{\text {reg }}(N)$. Within the space $\xi_{\text {reg }}(N) \subset \Sigma$, taking into account the identity $\mathbf{J}^{2}=\sigma^{2}+\omega^{2}$, which holds for regular spinors, it is always possible to associate a topological invariant for every regular state. Moreover, taking into account the usual mass dimension $3 / 2$ fermion, for which $\mathbf{J}$ represents the conserved current, the regarded topological invariant must be related to the electric charge. Before proceeding, let us make two parenthetical remarks. First, it is straightforward to realize that $\xi_{\text {reg }}$ may be naturally adapted for a lower dimension projection leading to elements as either $(\sigma, \mathbf{J}, 0,0,0)$ or $(0, \mathbf{J}, 0,0, \omega)$. Second, when we refer to the mass dimension of a given spinor, we mean the canonical mass dimension which shall be inherited by the quantum field from the dynamics respected by the expansion coefficients. Particular cases of the expansion coefficients are the objects treated here.

It is worth to emphasize that the space $\xi_{\text {reg }}(N)$ has a rich underlying geometric structure. Indeed, it consists not merely a of submanifold, but furthermore it manifests an intriguing structure arising from the monopole construction of the Hopf fibration $S^{1} \cdots S^{3} \rightarrow S^{2}$, where $S^{1}$ is homeomorphic to the Lie gauge group $U(1)$ of the electromagnetism [7]. Using a similar construction, the instanton is related to a principal bundle with structure Lie group SU(2), homeomorphic to the 3-sphere $S^{3}$. The instanton was described in Refs. [7,8] using the Hopf fibration $S^{3} \cdots S^{7} \rightarrow S^{4}$, in the context of the Witten monopole equations, by means of the bilinear 
covariants associated with regular spinor fields, under the Lounesto spinor field classification [16].

Let us make this point clear, working with a slightly different $\xi_{\text {reg }}(N)$ space, after which the general case shall be regarded. Regular spinor fields in either class 1 or class 2 in Lounesto's classification can be thought of as satisfying $\sigma=1$ without loss of generality, defining the manifold $S^{7}$, when the Dirac spinor field is classically described by an element of $\mathbb{C}^{4} \simeq \mathbb{H}^{2}$. Considering $\mathcal{C} \ell_{4}$ to be the Clifford algebra of the 4-dimensional Euclidean vector space $\mathbb{R}^{4}$, i.e., the algebra generated by the set of vectors $\left\{\mathrm{e}_{\mu}\right\}$, subject to the relations $\mathrm{e}_{\mu}^{2}=1$, and $\mathrm{e}_{\mu} \mathrm{e}_{\nu}+\mathrm{e}_{\nu} \mathrm{e}_{\mu}=0$, with $\mu=0,1,2,3$.

The quaternionic representation can be constructed by using Eq. (2), by $\mathrm{e}_{i} \mapsto\left[e_{i}\right]\left[e_{0}\right]$. One defines the observables of $\psi \in \mathbb{C}^{4}$ in a 4-dimensional Euclidean space by the following expressions:

$$
\begin{aligned}
\sigma & =\bar{\psi} \psi, \quad \omega=\bar{\psi} \mathrm{e}_{5} \psi, \quad J_{\mu}=\bar{\psi} \mathrm{e}_{\mu} \psi, \\
K_{\mu} & =i \bar{\psi} \mathrm{e}_{5} \mathrm{e}_{\mu} \psi, \quad S_{\mu \nu}=\bar{\psi}\left[\mathrm{e}_{\mu}, \mathrm{e}_{\nu}\right] \psi,
\end{aligned}
$$

where $\mathrm{e}_{5}=\mathrm{e}_{0} \mathrm{e}_{1} \mathrm{e}_{2} \mathrm{e}_{3}$. Note that the only different relations due to the Minkowski space case is the expression for $\omega$ (see Eq. (11a)). In fact, in the $\mathcal{C} \ell_{1,3}$ algebra one has $\mathrm{e}_{5}^{2}=-1$, whereas considering $\mathcal{C} \ell_{4}$ yields $\mathrm{e}_{5}^{2}=1$. In this way, the Fierz identities must be modified [7,15], yielding

$$
\begin{aligned}
\mathbf{J}^{2} & =\sigma^{2}-\omega^{2}, \quad \mathbf{J}^{2}=\mathbf{K}^{2}, \\
\mathbf{J} \wedge \mathbf{K} & =\left(\sigma-\mathrm{e}_{5} \omega\right) \mathbf{S}, \quad J^{\mu} K_{\mu}=\mathbf{J} \cdot \mathbf{K}=0 .
\end{aligned}
$$

In this context, the first Fierz identity (18) provides the expression $\mathbf{J}^{2}+\omega^{2}=1$ defining the 4 -sphere $S^{4}$, with coordinates $\left(J_{\mu}, \omega\right)$.

Now, taking into account Eqs. (6) and (11a) yields [7,8]

$\sigma=q_{1} \cdot q_{1}+q_{2} \cdot q_{2}, \quad \omega=2 \Re\left(q_{1}^{*} q_{2}\right)$,

$J_{0}=q_{1} \cdot q_{1}-q_{2} \cdot q_{2}, \quad J_{i}=2 \epsilon_{i}^{j k} \Re\left(q_{1}^{*} \mathrm{e}_{j} \mathrm{e}_{k} q_{2}\right)$,

for $i, j, k=1,2,3$ and $\epsilon_{i}{ }^{j k}$ is the Levi-Civita symbol. Hence, the representation in Eq. (17) reads [7,8]

$$
\begin{aligned}
\sigma & =\left|\psi_{1}\right|^{2}+\left|\psi_{2}\right|^{2}+\left|\psi_{3}\right|^{2}+\left|\psi_{4}\right|^{2}, \\
\omega & =2 \Re\left(\psi_{1} \psi_{3}^{*}+\psi_{2} \psi_{4}^{*}\right), \\
J_{0} & =\left|\psi_{1}\right|^{2}+\left|\psi_{2}\right|^{2}-\left|\psi_{3}\right|^{2}-\left|\psi_{4}\right|^{2}, \\
J_{1} & =2 \Im\left(\psi_{1} \psi_{4}^{*}+\psi_{2} \psi_{3}^{*}\right), \\
J_{2} & =2 \Re\left(\psi_{2} \psi_{3}^{*}-\psi_{1} \psi_{4}^{*}\right), \\
J_{3} & =2 \Im\left(\psi_{3} \psi_{1}^{*}+\psi_{2} \psi_{4}^{*}\right) .
\end{aligned}
$$

This important geometric feature reveals that the underlying geometry induced by spinors classes can further point to more structures. Indeed, the following proposition regards the topological invariants associated to regular spinors.

Proposition 1 Let $\xi_{\text {reg }}(N)$ be the space consisting of regular spinors. Then

$\pi_{\left[\operatorname{dim}\left(\xi_{\operatorname{reg}}(N)\right)-1\right]}\left(\xi_{\text {reg }}(N)\right)=n \in \mathbb{Z}$, where $\pi_{i}(R)$ stands for the $i^{\text {th }}$-homotopy group of a given $R$ space. In the case of a mass dimension $3 / 2$ fermion described by the regular spinor, the topological invariant is associated to the electric charge.

Proof The first assertion directly follows from the above discussion. In fact, a regular spinor is an element of $\xi_{\text {reg }}(N)$ and therefore there exist just two possibilities:

- $\operatorname{dim} \xi_{\text {reg }}(N)=3$, and the spinor belongs to class 1 of Lounesto's classification. In this case the point $(0,0,0) \in$ $\xi_{\text {reg }}(N)$ cannot be attained, implying that $\pi_{2}\left(\xi_{\text {reg }}(N)\right)=$ $n \in \mathbb{Z}$

- $\operatorname{dim} \xi_{\text {reg }}(N)=2$, and thus the spinor belongs to either class 2 or class 3 of Lounesto's classification. This is the case when $\pi_{1}\left(\xi_{\text {reg }}(N)\right)=n \in \mathbb{Z}$.

In general, $\mathbf{J}$ is not necessary related to the conserved current associated to a given fermion. Nevertheless, when it doeswhich is the case for mass dimension $3 / 2$ fermions-then the conserved charge is the electric charge itself. Since a vanishing charge is forbidden in these situations, one has the physical counterpart of the above topological constraint. Indeed the $(0,0,0)$ point for regular spinors can never be reached.

As readily verified, in the case of the 3-dimensional $\xi_{\text {reg }}(N)$ space the conserved current may also be seen as the generator of cohomology, for $H^{1}\left(\xi_{\text {reg }}(N)\right) \simeq H^{1}\left(\mathbb{R}^{2} \backslash\{0\}\right)$, and the usual closed form

$$
\frac{\sigma \mathrm{d} \omega-\omega \mathrm{d} \sigma}{\sigma^{2}+\omega^{2}},
$$

is not everywhere exact. The usefulness of the representation space construction is now evident. By treating physical spinor (states) as points of a given space, constrained by the algebraic bilinear relations, it is possible to work in the interplay of topology, multivector algebra, and physics. As a matter of fact, the very existence of a relativistic spinor is related to a topological invariant in the representation space.

Nevertheless, one shall not be so optimistic just by looking at the example just studied, since we were dealing only with a projection. The $\Sigma$ space, where not only regular spinors are taken into account, is certainly very difficult to analyze. There are, however, some interesting points that we shall report on the study of $\Sigma$ in its general form. In fact, Lounesto's classification provides six classes of spinors, wherein a continuous path in the representation space allows access to different configuration states. Let us make this idea clearer and more precise.

All spinors in an arbitrary class are connected by a simple rescaling. From the point of view of elements in $\Sigma(N)$, two different elements $\Psi^{\prime}$ and $\Psi$ are connected by an usual transformation along the same class by $\Psi^{\prime}=S \Psi$. In this context, 
it is possible to assert, in a manner akin to Wigner [30,31], the following proposition.

Proposition 2 Let $D_{\lambda}$ be a 1-parameter infinitesimal operator acting on the spinor space of a given class according to Lounesto's classification. Suppose that it is an homomorphism, $D_{\lambda} D_{\lambda^{\prime}}=D_{\lambda+\lambda^{\prime}}$, with $\lambda \in \mathbb{R}$. If there exists a physical state on which the application of $D_{\lambda}$ is well defined, then there exists a dense set of such states in the respective class, with respect to Lounesto's classification.

Proof If the application of $D_{\lambda}$ is well defined for a given

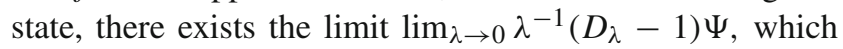
implies that $\lim _{\lambda \rightarrow 0} \lambda^{-1}\left(D_{\lambda} S^{-1} S-S^{-1} S\right) \Psi$. Since the rescaling commutes with $D_{\lambda}$, it follows that there exists the limit $\lim _{\lambda \rightarrow 0} \lambda^{-1}\left(D_{\lambda}-1\right) S \Psi$. Hence, within an arbitrary but fixed class in Lounesto's classification, it is possible to operate with infinitesimal operators in a rather usual way. Moreover, in view of the above result, physical spinors are indeed points of $\Sigma(N)$.

It is important to remark that an arbitrary class in Lounesto's classification is invariant under $S$. It is furthermore possible, however, to connect two different classes by algebraic transformation. More specifically, it was shown in Ref. [32] that there exists a subset of spinors in class 1, 2, and 3 which can be mapped into a subclass of class 5 spinors. Let us denote this transformation, between different classes, by $S_{C}$. It turns out that det $S_{C} \neq 0$ [32]. Hence, the alluded algebraic bridge, in a manner of speaking, is also dense. In fact, as far as we restrict ourselves to the subset of states which can be mapped, the proof of Proposition 2 holds, in this switching class case.

A given algebraic bridge, however, is not always necessarily well behaved. In the sequel we give a (counter-) example, presenting a mapping between a subset of spinors in class 1,2 , and 3 , and a subset in class 4 , which is neither Hermitian nor invertible. As one shall see, this example is quite severe as regards the constraints it imposes, but the possibility of a more manageable mapping is not discarded. It is worth to stressing that the mapping, from regular spinors to class 4 spinors, is chosen as a particular case. Class 4 spinors are understood as the most unvoiced class in Lounesto's classification, having just a rare single example in the literature [18] as a physical solution of the Dirac equation in a Riemann-Cartan Bianchi-I, $f(R)$, background. Lounesto describes such class as the only one that, at that time, had not corresponded to any type of spinor already found in Nature [3]. Except for such solution, neither other types of flagdipole spinors nor their respective dynamics as well have been found, yet. The algebraic mapping between regular and class 4 spinors can be parenthetically seen, then, as an attempt to put forward a bottom-up approach, embracing flag-dipoles spinors into the standard setup of high energy physics.
We start by introducing a matrix $M=\left(\begin{array}{ll}\mathbb{M}_{11} & \mathbb{M}_{12} \\ \mathbb{M}_{21} & \mathbb{M}_{22}\end{array}\right) \in$ $\mathcal{M}(4, \mathbb{C})$, defining the mapping:

$$
\begin{aligned}
M: \mathcal{D} & \rightarrow \mathcal{T}_{4} \\
\Phi_{D} & \mapsto \Psi_{4}=M \Phi_{D},
\end{aligned}
$$

where $\mathcal{D}$ and $\mathcal{T}_{4}$ stands for the sets comprising regular and flag-dipole spinors, respectively. The formalism is clearly representation-independent, however, the Weyl representation $\gamma_{0}=\left(\begin{array}{ll}\mathbb{O} & \mathbb{I} \\ \mathbb{I} & \mathbb{O}\end{array}\right), \gamma_{k}=\left(\begin{array}{cc}\mathbb{O} & \sigma_{k} \\ -\sigma_{k} & \mathbb{O}\end{array}\right)$ shall be used, $\sigma_{k}$ being the usual Pauli matrices, to fix the notation hereby.

According to the Lounesto spinor classification, using the mapping defined in (22), class 4 spinors satisfy

$\sigma=\Phi_{D}^{\dagger} M^{\dagger} \gamma_{0} M \Phi_{D}=0, \quad \omega=-\Phi_{D}^{\dagger} M^{\dagger} \gamma_{123} M \Phi_{D}=0$.

Let us investigate here the constraints exclusively on the $M$ matrix. The conditions (23) imply

$M^{\dagger} \gamma_{0} M=0, \quad M^{\dagger} \gamma_{123} M=0$,

which, in the Weyl representation, yield

$$
\begin{aligned}
& \pm \mathbb{M}_{11}^{\dagger} \mathbb{M}_{21}+\mathbb{M}_{21}^{\dagger} \mathbb{M}_{11}=\mathbb{O}= \pm \mathbb{M}_{11}^{\dagger} \mathbb{M}_{22}+\mathbb{M}_{21}^{\dagger} \mathbb{M}_{12} \\
& \pm \mathbb{M}_{12}^{\dagger} \mathbb{M}_{21}+\mathbb{M}_{22}^{\dagger} \mathbb{M}_{11}=\mathbb{O}= \pm \mathbb{M}_{12}^{\dagger} \mathbb{M}_{22}+\mathbb{M}_{22}^{\dagger} \mathbb{M}_{12}
\end{aligned}
$$

The system (25)-(26) is satisfied when

$\mathbb{M}_{11}^{\dagger} \mathbb{M}_{21}=\mathbb{M}_{11}^{\dagger} \mathbb{M}_{22}=\mathbb{M}_{12}^{\dagger} \mathbb{M}_{21}=\mathbb{M}_{12}^{\dagger} \mathbb{M}_{22}=\mathbb{O}$.

Therefore, by considering a general representation,

$\mathbb{M}_{11}=\left(\begin{array}{ll}m_{11} & m_{12} \\ m_{21} & m_{22}\end{array}\right), \quad \mathbb{M}_{12}=\left(\begin{array}{ll}m_{13} & m_{14} \\ m_{23} & m_{24}\end{array}\right)$,

$\mathbb{M}_{21}=\left(\begin{array}{ll}m_{31} & m_{32} \\ m_{41} & m_{42}\end{array}\right), \quad \mathbb{M}_{22}=\left(\begin{array}{ll}m_{33} & m_{34} \\ m_{43} & m_{44}\end{array}\right)$,

it is possible to rewrite

$M=\left(\begin{array}{cccc}m_{11} & m_{12} & m_{13} & m_{14} \\ \frac{m_{11} m_{22}}{m_{12}} & m_{22} & \frac{m_{13} m_{22}}{m_{12}} & \frac{m_{14} m_{22}}{m_{12}} \\ \frac{-m_{22}^{*} m_{41}}{m_{12}^{*}} & \frac{-m_{22}^{*} m_{42}}{m_{12}^{*}} & \frac{-m_{22}^{*} m_{43}}{m_{12}^{*}} & \frac{-m_{22}^{*} m_{44}}{m_{12}} \\ m_{41} & m_{42} & m_{43} & m_{44}\end{array}\right)$.

The $M$ matrix is responsible for performing the mapping between regular and class 4 spinors. ${ }^{1}$ There are, however, important restrictions on such a mapping which must be highlighted.

Firstly, the mapping performed cannot occur from class 4 to regular spinors. In fact, supposing the existence of $\stackrel{M}{M}$

\footnotetext{
1 A simple, but tedious calculation shows that the other bilinears behave in such a way that the mapping (28) works well, ensuring a final class 4 spinor.
} 
such that $\stackrel{\circ}{M} \Psi_{4}=\Phi_{D}$, then $M \stackrel{M}{M} \Psi_{4}=M \Psi_{4}$, leading to $\stackrel{\circ}{M}=M^{-1}$. Nevertheless, as it can be explicitly calculated from (28), $\operatorname{det} M=0$ and there is no such a $\stackrel{M}{M}$ matrix. Secondly, the mapping (28) cannot be Hermitian. Indeed, the requirement $M=M^{\dagger}$ yields

$m_{11}=m_{11}^{*}, \quad m_{12}=m_{12}^{*}, \quad m_{22}=m_{22}^{*}, \quad m_{14}=m_{41}^{*}$,

$m_{13}=\frac{-m_{22} m_{14}}{m_{12}}=-m_{42}^{*}, \quad m_{44}=\frac{-m_{12} m_{43}}{m_{22}}$.

Nevertheless, the other bilinear invariants do not behave as the ones for class 4 spinors. In fact, it can be verified that $\mathbf{K}=0=\mathbf{S}$ for this case, rendering a spinor different from a class 4 one and then the hermiticity is forbidden.

It is significant to stress that the above mapping was performed using class 1 regular spinors, as it is clear from (22) and (23). Nevertheless, as far as we implement the additional constraints coming from class 2 and 3 Dirac-like spinors, the final form of the bilinear invariants are slightly modified, but the net result is the same. While property one is useful in the study of possible information as regards the representation space (in the context of Proposition 2), the study of the hermiticity property may be relevant in a quantum mechanical context.

The counter-example just studied indicates an elaborated representation space, whose non-triviality deserves further exploration. It must be once again emphasized, however, that the constraints (24) are too restrictive, since they extend the kernel of the transformation to the whole $(\sigma, \omega)$-plane.

We would like to finalize this section by pointing out three new classes of spinors, beyond the Lounesto's classification in Table 1, which also reside in the spinor representation space. These spinors were obtained in the operatorial and algebraic form in Ref. [33], having, by construction, $\mathbf{J}=0$. Therefore, their dynamics cannot be described by the Dirac operator. The nontrivial topology of the representation space, as already remarked, is inherited from the constraints imposed by the FPK identities. For the sector of $\Sigma(N)$ comprised by regular spinors, $\mathbf{J}$ is the generator of cohomology and cannot vanish. The sector of $\Sigma(N)$ encompassing singular spinors, nevertheless, may also accommodate the spinors of [33]. Notice that a vanishing $\mathbf{J}$ does not lead to a contradiction, and the FPK identities still hold in this case. Hence, these spinors are also physical in the sense previously discussed. The spinors found in [33], assuming that $\mathbf{J}=0$, may be called pole (only $\mathbf{K} \neq 0$ ), flag (only $\mathbf{S} \neq 0$ ), or flag-pole. ${ }^{2}$ They live in a special subspace of $\Sigma(N)$ whose topology also deserves further attention.

${ }^{2}$ It is worth mentioning that these flag-poles are essentially different of the standard flag-poles characterized by the flag $\mathbf{S} \neq 0$ ) and the pole $(\mathbf{J} \neq 0)$, since in this case $(\mathbf{K}=0)[3]$.

\section{Passing through spinor classes: a natural dynamical interplay}

Apart from the mentioned algebraic bridges, and the counterexample previously examined, we shall study a physical system whose dynamics provides an interesting interface to the abstract idea of a path changing classes in the representation space. This section is somewhat disconnected from the mathematical scope we intended to attribute to this formal paper, nevertheless the physical discussion demands such a change.

Coupling a self-interacting Dirac fermion to gravity is ruled by an action related to the Einstein-Dirac system, which reads

$$
\begin{aligned}
S= & \int d^{4} x \sqrt{-g}\left[M_{p}^{2}(2 R-\Lambda)+\frac{i}{2}\left(\left(D_{\mu} \bar{\psi}\right) \gamma^{\mu}\right.\right. \\
& \left.\left.-\bar{\psi} \gamma^{\mu} D_{\mu}\right) \psi-m \bar{\psi} \psi+\frac{\lambda}{2}(\bar{\psi} \psi)^{2}\right],
\end{aligned}
$$

where $\Lambda$ denotes the cosmological constant, $m$ represents the mass of the spinor field and the Planck mass $M_{p}^{-2}=8 \pi G$ shall be 2 , setting $16 \pi G=1 ; \lambda$ denotes the fermion (effective) self-interaction coupling constant. In fact such a term is not perturbatively renormalizable, and therefore it must be understood as an effective coupling, being suppressed by powers of a fundamental scale. Besides, the covariant derivative read $[18,19]$

$D_{\mu} \psi=\left(\partial_{\mu}+\frac{i}{2}\left(\Gamma_{\mu \nu}^{\rho} e_{a \rho}-\partial_{\mu} e_{a v}\right) e_{b}^{\nu} \gamma^{a b}\right) \psi$,

where the $\Gamma_{\mu \nu}^{\rho}$ are the Christoffel symbols and the tetrad $e_{a \mu}$ satisfy the relations $g_{\mu \nu}=\eta^{a b} e_{a \mu} e_{b v}$ and $\gamma^{\mu}=\gamma^{a} e_{a}^{\mu}$. The equations of motion that can be derived from the action (30) are

$$
\begin{aligned}
\gamma^{\mu} D_{\mu} \psi+m \psi= & \lambda \bar{\psi} \psi \\
-4 i\left(2 R_{\mu \nu}-\Lambda g_{\mu \nu}\right)= & {\left[\bar{\psi} \gamma_{(\mu} D_{\nu)} \psi-D_{(\mu} \bar{\psi} \gamma_{\nu)} \psi\right] } \\
& +g_{\mu \nu}\left(m \bar{\psi} \psi-\frac{1}{2} \lambda(\bar{\psi} \psi)^{2}\right)
\end{aligned}
$$

Using spherical coordinates, axisymmetric spacetimes $M$ can have an ansatz of type [20]

$$
\begin{aligned}
\mathrm{d} s^{2}= & \left(\dot{g}_{z}^{2}-g_{t}^{2}\right) \mathrm{d} t^{2}+g_{r}^{2} \mathrm{~d} r^{2} \\
& -2\left(g_{t} g_{z}+g_{p} g_{z}\right) \mathrm{d} t \mathrm{~d} \phi+\left(g_{p}^{2}-g_{t}^{2} g_{z}^{2}\right) \mathrm{d} \phi^{2}+g_{y}^{2} \mathrm{~d} \theta^{2} .
\end{aligned}
$$

Besides, closed time-like curves are precluded when the condition $\left(g_{p}^{2}-g_{t}^{2} g_{z}^{2}\right)>0$ is imposed. The vierbeins can be expressed as

$e^{0}=g_{t}\left(\mathrm{~d} t+g_{z} \mathrm{~d} \phi\right), \quad e^{1}=g_{r} \mathrm{~d} r$,

$e^{2}=g_{y} \mathrm{~d} \theta, \quad e^{3}=g_{p} \mathrm{~d} \phi-\stackrel{\circ}{z}_{z} \mathrm{~d} t$.

The spinor field $\psi=\left(\psi_{1}(x), \psi_{2}(x), \psi_{3}(x), \psi_{4}(x)\right)^{\top}$, where $\psi_{i}=\psi_{a 1}+i \psi_{a 2}: M \rightarrow \mathbb{C}$, can be used to solve (32) 
and (33), for asymptotically flat spacetimes. The ansätzes are Maclaurin series in the variable $r^{-j}$, for the metric components, given by

$g_{t}=1+\sum_{j=1}^{\infty} \frac{t_{j}}{r^{j}}, \quad g_{z}=\sum_{j=1}^{\infty} \frac{z_{j}}{r^{j}}, \quad g_{r}=1+\sum_{j=1}^{\infty} \frac{r_{j}}{r^{j}}$,

$g_{y}=r+\sum_{j=0}^{\infty} \frac{y_{j}}{r^{j}}, \quad g_{p}=\sin \theta\left(r+\sum_{j=0}^{\infty} \frac{p_{j}}{r^{j}}\right)$,

$\psi_{i}=\sum_{j=1}^{\infty} \frac{a_{i j}+i b_{i j}}{r^{j}}, \quad$ for $i=1, \ldots, 4$

The involved functions are assumed to be functions of $r, \theta$ and $t$ and are expanded with respect to $r$, up to order $\mathcal{O}\left(r^{-4}\right)$, considering one more order than Ref. [19] and the self-interaction in Eq. (30). Such expansions in Eqs. (36)-(38) ensure that the limit yields an asymptotically flat metric.

Given (37) and (38), the leading contribution to (32) is of the order $\mathcal{O}\left(\frac{1}{r}\right)$. Assuming light-cone like coordinates $r^{ \pm}=$ $r \pm c t$, the equations are given by

$$
\begin{array}{ll}
m b_{31}-\partial_{-} a_{11}=0, & m a_{31}+\partial_{-} b_{11}=0, \\
m b_{41}+\partial_{+} a_{21}=0, & m a_{41}-\partial_{+} b_{21}=0, \\
m b_{11}+\partial_{+} a_{31}=0, & m a_{11}-\partial_{+} b_{31}=0, \\
m b_{21}-\partial_{-} a_{41}=0, & m a_{21}+\partial_{-} b_{41}=0 .
\end{array}
$$

These equations can be completely solved by

$$
\begin{aligned}
a_{i 1}= & {\left[a_{i 1 a}(\theta) \cos (k r)+a_{i 1 b}(\theta) \sin (k r)\right] \cos (\omega t) } \\
& +\left[a_{i 1 c}(\theta) \cos (k r)+a_{i 1 d}(\theta) \sin (k r)\right] \sin (\omega t),
\end{aligned}
$$

where $\omega^{2}=k^{2}+m^{2}$. The $b_{i 1}$ coefficients in Eq. (38) are solved in terms of $a_{i 1}$. The leading contribution to (33) is of the order $\mathcal{O}(r)$. The equations are wave equations for $y_{0}$ and $p_{0}$, whose solutions read functions that can be generally expressed as the wave $c_{0}=c_{0}(\theta)+c_{01}(\theta, r-t)+c_{02}(\theta, r+$ $t$ ), for the two first coefficients $y_{0}$ and $p_{0}$ in the series (37) [19]. The metric coefficients can be solved as

$$
\begin{aligned}
g_{t}= & 1-\frac{\mathrm{A}^{2} \omega \cos \theta}{4 k m r}, \\
g_{r}= & 1-\frac{\mathrm{A}^{2} \omega^{3} \cos \theta}{4 k^{3} m r}+\frac{\mathrm{A}^{2} k \sin [2(\omega t-\vartheta)] \sin \theta}{4 \omega^{2} m r^{2}} \\
& -\lambda \frac{\mathrm{A}^{2} k^{2} \sin (3 \omega t-3 \vartheta) \sin 2 \theta}{16 \omega^{4} m^{2} r^{3} b}, \\
g_{y}= & +\frac{\mathrm{A}^{2}}{4 k^{3} m} \omega\left(2 k^{2}+\omega^{2}\right) \cos \theta \\
& -\frac{\mathrm{A}^{2} k}{4 \omega^{2} m r} \sin [2(\omega t-\vartheta)] \sin \theta \\
& +\frac{\mathrm{A}^{4}\left(2 k^{2}-\omega^{2}\right)}{16 k^{2} \omega m^{2} r^{2}} \sin [2(\omega t-\vartheta)] \sin 2 \theta,
\end{aligned}
$$

$$
\begin{aligned}
& +\lambda \frac{\mathrm{A}^{6}\left(4 k^{2}+\omega^{2}\right)}{64 k^{4} \omega m^{3} r^{3}} \sin [2(\omega t-\vartheta)] \sin 3 \theta \\
g_{z}= & \frac{\mathrm{A}^{2}}{8 k^{2} m^{2} r^{2}}\left[6 \omega \cos (2 k r) \sin ^{2} \theta\right. \\
& +k \sin (2 k r) \sin [2(\omega t-\vartheta)] \sin 2 \theta] \\
& -\frac{\mathrm{A}^{2} \omega \sin (2 k r) \sin ^{2} \theta}{2 k m^{2} r}
\end{aligned}
$$

and $g_{p}=\sin \theta g_{y}$. This metric can be led to the case where there is no self-interaction, considering terms to order $r^{-3}$, as in Ref. [19]. Hence, the spinor field reads

$$
\begin{aligned}
\psi_{1 a}= & \mathcal{A}_{+}\left[\left(1-\frac{\mathrm{A}_{+}}{r}\right) \cos (\omega t+k r+\vartheta)\right. \\
& \left.+\AA_{+}\left(1+\lambda \AA_{+}\right) \sin (\omega t-k r+\vartheta)\right], \\
\psi_{1 b}= & \mathcal{A}_{-}\left[\left(1-\frac{\mathrm{A}_{-}}{r}\right) \cos (\omega t-k r+\vartheta)\right. \\
& \left.+\AA_{-}\left(1-\lambda \AA_{-}\right) \sin (\omega t+k r+\vartheta)\right],
\end{aligned}
$$

$\psi_{2 a}=\mathcal{A}_{+}\left[\left(1-\frac{\mathrm{A}_{+}}{r}\right) \cos (\omega t-k r+\vartheta)\right.$

$$
\left.+\AA_{+}\left(1+\lambda \AA_{+}\right) \sin (\omega t+k r+\vartheta)\right],
$$

$\psi_{2 b}=\mathcal{A}_{-}\left[\left(1-\frac{\mathrm{A}_{-}}{r}\right) \cos (\omega t+k r+\vartheta)\right.$

$$
\left.+\AA_{-}\left(1-\lambda \AA_{-}\right) \sin (\omega t-k r+\vartheta)\right] \text {, }
$$

$\psi_{3 a}=-\mathcal{A}_{+}\left[\left(1-\frac{\mathrm{A}_{+}}{r}\right) \sin (\omega t-k r+\vartheta)\right.$

$$
\left.+\AA_{+}\left(1+\lambda \AA_{+}\right) \cos (\omega t+k r+\vartheta)\right] \text {, }
$$

$\psi_{3 b}=\mathcal{A}_{-}\left[\left(1-\frac{\mathrm{A}_{-}}{r}\right) \sin (\omega t-k r+\vartheta)\right.$

$$
\left.+\AA_{-}\left(1-\lambda \AA_{-}\right) \cos (\omega t-k r+\vartheta)\right] \text {, }
$$

$$
\begin{aligned}
\psi_{4 a}= & -\mathcal{A}_{+}\left[\left(1-\frac{\mathrm{A}_{+}}{r}\right) \sin (\omega t-k r+\vartheta)\right. \\
& \left.+\AA_{+}\left(1+\lambda \AA_{+}\right) \cos (\omega t-k r+\vartheta)\right], \\
\psi_{4 b}= & \mathcal{A}_{-}\left[\left(1-\frac{\mathrm{A}_{-}}{r}\right) \sin (\omega t-k r+\vartheta)\right. \\
& \left.+\AA_{-}\left(1-\lambda \AA_{-}\right) \cos (\omega t+k r+\vartheta)\right],
\end{aligned}
$$

where $\mathcal{A}_{ \pm}=\frac{\mathrm{A} \sqrt{\sin \theta}}{r \sqrt{\omega \mp k}}, \mathrm{~A}_{ \pm}=\frac{\mathrm{A}^{2}(4 k \mp \omega) \cos \theta}{8 k^{2} m r}$, and $\AA_{ \pm}=$ $\frac{(2 k \pm \omega) \cot \theta}{4 k(\omega \pm k) r}$, where $\mathrm{A}$ is a constant and $\vartheta$ is a phase. 
The current density of the spinor field, $J^{\mu}=\bar{\psi} \gamma^{\mu} \psi$ implies $D_{\mu} J^{\mu}=0$. Turning off the spinor self-interaction hereon, for the sake of simplicity, the metric (34) with components (42) yields an approximate time-like Killing vector $\xi=\partial_{t}-\frac{1}{r} \partial_{t} y_{1} \partial_{r}$ at the spatial infinity [19]. Besides, the Ricci scalar, computed with respect to the metric components Eq. (42), reads $R=\frac{2 \mathrm{~A}^{2} k \sin [2(\omega t+\vartheta)] \sin \theta}{m r^{2}}$, resulting in a curvature that oscillates with a frequency of $2 \omega$, whose sign varies with such a frequency. Moreover, given $\epsilon_{\mu \nu \rho \sigma}$ the Levi-Civita tensor multiplied by $\sqrt{|g|} / 2$, the dual of the exterior product between the Killing vector and the spinor intrinsic angular momentum, $S^{\mu} \equiv i \epsilon^{\mu \nu \sigma \rho} S_{\sigma \rho} \xi_{v}=0$, vanishes.

Moreover, for values of the time variable

$$
\begin{aligned}
t & \sim \omega^{-1}\left[k r+\vartheta-\tan ^{-1}\left(\frac{D}{4 k-2 \omega}\right.\right. \\
& \left.\left.-\frac{2 k m(2 k-\omega) \cot \theta}{B}\right)\right], \quad c_{1} \in \mathbb{Z},
\end{aligned}
$$

where

$$
\begin{aligned}
B^{2}= & 16 C^{-1}\left(r^{2} m^{2} k^{6}-8 r^{2} m^{2} \omega k^{5}-4 \mathrm{~A}^{2} r m \omega \cos \theta k^{5}\right. \\
& +4 r^{2} m^{2} \omega^{2} k^{4}+\mathrm{A}^{4} \omega^{2} \cos ^{2} \theta k^{4}+m^{2} \cot ^{2} \theta k^{4} \\
& -3 \mathrm{~A}^{4} \omega^{3} \cos ^{2} \theta k^{3} / 2-m^{2} \omega \cot ^{2} \theta k^{3} \\
& -2 \mathrm{~A}^{2} r m \omega^{3} \cos \theta k^{3}+\mathrm{A}^{4} \omega^{4} \cos ^{2} \theta k^{2} \\
& +4 m^{2} \omega^{2} \cot ^{2} \theta k^{2}-\mathrm{A}^{2} r m \omega^{4} \cos \theta k^{2} \\
& +3 \mathrm{~A}^{4} \omega^{5} \cos ^{2} \theta k / 5+\mathrm{A}^{4} \omega^{6} \cos ^{2} \theta \\
& \left.+7 \mathrm{~A}^{2} r m \omega^{2} \cos \theta k^{4}-\pi c_{1} / 8\right),
\end{aligned}
$$

where $c_{1} \in \mathbb{Z}$ and

$$
\begin{aligned}
D= & {\left[32 r m k^{4}-48 r m \omega k^{3}+16 r m \omega^{2} k^{2}\right.} \\
& \left.+20 \mathrm{~A}^{2} \omega^{2} \cos \theta k^{2}-2 \mathrm{~A}^{2} \omega^{3} \cos \theta k-2 \mathrm{~A}^{2} \omega^{4} \cos \theta\right] \\
C^{2}= & 4\left(r^{2} m^{2} k^{6}-8 r^{2} m^{2} \omega k^{5}-4 \mathrm{~A}^{2} r m \omega \cos \theta k^{5}\right. \\
& +4 r^{2} m^{2} \omega^{2} k^{4}+\mathrm{A}^{4} \omega^{2} \cos ^{2} \theta k^{4}+m^{2} \cot ^{2} \theta k^{4} \\
& +7 \mathrm{~A}^{2} r m \omega^{2} \cos \theta k^{4}-3 \mathrm{~A}^{4} \omega^{3} \cos ^{2} \theta k^{3} / 2 \\
& \left.-m^{2} \omega \cot ^{2} \theta k^{3}-2 \mathrm{~A}^{2} r m \omega^{3} \cos \theta k^{3}\right) \\
& +\mathrm{A}^{4} \omega^{4} \cos ^{2} \theta k^{2}+4 m^{2} \omega^{2} \cot ^{2} \theta k^{2} \\
& -16 \mathrm{~A}^{2} r m \omega^{4} \cos \theta k^{2}+6 \mathrm{~A}^{4} \omega^{5} \cos ^{2} \theta k \\
& +\mathrm{A}^{4} \omega^{6} \cos ^{2} \theta
\end{aligned}
$$

the spinor field $\psi$ is a flag-dipole one, since the components of the intrinsic angular momentum $S_{\mu \nu}=\bar{\psi}\left[\gamma^{\mu}, \gamma^{\nu}\right] \psi$ vanish, together also with $\sigma=\bar{\psi} \psi$ and $\omega=\bar{\psi} \gamma_{5} \psi$. It dynamically implements the formalism constructed in the previous section. In fact, such a dynamical setup there are changes of the regular spinors of type 1 into singular spinors of type 4 , resulting in a physical couterpart to the mapping (22).

\section{Concluding remarks}

The formalization of a spinor representation space, whose points can be faced as physical spinors, has been constructed. These spinors have been shown to behave as elements of dense paths of the representations space which, in view of the FPK identities, perform highly topologically constrained subsets. Some of these subsets have topological properties intrinsically connected to physical relevant quantities. The representation space shows itself as an adequate tool to explore dynamics and interactions usually by means of using infinitesimal operators.

It should be emphasized that along this work we took advantage of dealing with spinors as elements of $\mathcal{C} \ell_{1,3} \frac{1}{2}(1+$ $\left.e_{0}\right)$ in Sect. 2. Similar constraints in the representation space, coming from the FPK identities in the $\mathrm{Cl}_{4}$ isomorphic case, are expected. However, our main interest here is the study of the representation space taking into account the Clifford algebra constructed upon the Minkowski space.

In showing that type 4 spinors cannot be led into regular ones, we asserted that the mapping connecting different physical spinors-spinors of different sectors of $\Sigma(N)$, belonging to different classes. However, no reference has been made to quantum mechanics. It is time to elaborate this a little further. The very possibility of crossing over different classes, by means of a well-defined algebraic transformation connecting different sectors of $\Sigma(N)$ could, in principle, be related to some type of swapping spinor class due to a specific physical process. In fact, bearing in mind the existence of a dense set in between different classes in the light of proposition two, this switching could be performed by a specific (unknown) scattering matrix modeling the physical process. Apart from unitarity concerns, ${ }^{3}$ it is difficult to envisage how this proposed process can duplicate the helicity states in going from regular spinors to type 5 spinors, these last spinors with known dual property helicity. Perhaps, and here we are entering the fancy ground of speculation, a comprehensive transformation performed in the quantum operator as a whole may give rise to more precision to and enlighten the formal aspect of this possible swapping. It turns out, however, that the physical process would still be lacking. On the other hand, we have provided a mapping which is neither invertible nor Hermitian, evincing the high degree of topological constraint presented on the representation space. Further investigation on the algebraic/topological relationship concerning singular spinors are under current investigation.

From the physical point of view, changing spinor classes is essentially a change of physical observables. The dynamical setup as a procedure to change spinor classes was implemented for an axisymmetric spacetime that is generated by

\footnotetext{
3 Typically, the $S_{C}$ matrix have enough symmetry to be recast into a specific form allowing for an unitary scattering process.
} 
the self-gravity of the spinor here studied, satisfying the equations of motion derived from an Einstein-Hilbert action with cosmological constant coupled to a Dirac system with selfinteraction.

Acknowledgements JMHS thanks to CNPq (304629/2015-4; 445385/ 2014-6) for partial financial support. CHCV thanks to PNPD-CAPES for financial support. RJBR thanks to CAPES for financial support, and RdR is grateful to CNPq (Grant No. 303293/2015-2), and to FAPESP (Grant No. 2015/10270-0), for partial financial support.

Open Access This article is distributed under the terms of the Creative Commons Attribution 4.0 International License (http://creativecomm ons.org/licenses/by/4.0/), which permits unrestricted use, distribution, and reproduction in any medium, provided you give appropriate credit to the original author(s) and the source, provide a link to the Creative Commons license, and indicate if changes were made.

Funded by SCOAP ${ }^{3}$.

\section{References}

1. S.L. Altmann, Rotations, Quaternions, and Double Groups (Clarendon Press, Oxford, 1986)

2. S. Weinberg, The Quantum Theory of Fields, I: Foundations (Cambridge University Press, New York, 2005)

3. P. Lounesto, Clifford Algebras and Spinors, 2nd edn. (Cambridge University Press, Cambridge, 2001)

4. J.P. Crawford, J. Math. Phys. 26, 1439 (1985)

5. R.A. Mosna, W.A. Rodrigues Jr., J. Math. Phys. 45, 2945 (2004)

6. W.A. Rodrigues Jr., J. Math. Phys. 45, 2908 (2004)

7. J. Vaz Jr., Construction of monopoles and instantons using spinors and the inversion theorem, in Clifford Alg. Appl. in Math. Phys, ed. by V. Dietrich, et al. (Kluwer, Dordrecht, 1998), pp. 401-421

8. J. Vaz Jr., Clifford algebras and Witten's monopole equations, in Geometry, Topology and Physics, ed. by B. Apanasov, S. Bradlow, W.A. Rodrigues, K. Uhlenbeck (Verlag, Berlin, 1997), pp. 277-300
9. R. Abłamowicz, I. Gonçalves, R. da Rocha, J. Math. Phys. 55, $103501(2014)$

10. J. Vaz, R. da Rocha, An Introduction to Clifford Algebras and Spinors (Oxford University Press, Oxford, 2016)

11. V.L. Figueiredo, E. Capelas de Oliveira, W.A. Rodrigues, Int. J. Theor. Phys. 29, 371 (1990)

12. D. Hestenes, J. Math. Phys. 8, 798 (1967)

13. J.D. Bjorken, S.D. Drell, Relativistic Quantum Mechanics (McGraw Hill, New York, 1964)

14. P.R. Holland, Found. Phys. 16, 701 (1986)

15. R.A. Mosna, J. Vaz, Phys. Lett. A 315, 418 (2003)

16. R. da Rocha, J.M. Hoff da Silva, Adv. Appl. Clifford Algebras 20 , 847 (2010)

17. C.H. Coronado Villalobos, R.J. Bueno Rogerio, EPL 116, 60007 (2016)

18. R. da Rocha, L. Fabbri, J. Hoff da Silva, R. Cavalcanti, J. Neto, J. Math. Phys. 54, 102505 (2013)

19. J. Mei, Phys. Lett. B 701, 279 (2011)

20. J. Mei, Gen. Relat. Gravit. 44, 2191 (2012)

21. J.M. Hoff da Silva, C.H. Coronado Villalobos, R.J. Bueno Rogerio, E. Scatena, Eur. Phys. J. C 76, 563 (2016)

22. C.Y. Lee, Phys. Lett. B 760, 164 (2016)

23. J.M. Hoff da Silva, R. da Rocha, Phys. Lett. B 718, 1519 (2013)

24. L. Fabbri, Phys. Lett. B 704, 255 (2011)

25. D.V. Ahluwalia, D. Grumiller, JCAP 0507, 012 (2005)

26. L. Bonora, K.P.S. de Brito, R. da Rocha, JHEP 1502, 069 (2015)

27. R. da Rocha, A.E. Bernardini, J.M. Hoff da Silva, JHEP 1104, 110 (2011)

28. R.T. Cavalcanti, Int. J. Mod. Phys. D 23, 1444002 (2014)

29. L. Fabbri, Int. J. Geom. Meth. Mod. Phys. 13, 1650078 (2016)

30. E.P. Wigner, Ann. Math. 40, 149 (1939)

31. E.P. Wigner, Nucl. Phys. Proc. Suppl. 6, 9 (1989)

32. R. da Rocha, J.M. Hoff da Silva, J. Math. Phys. 48, 123517 (2007)

33. C.H. Coronado Villalobos, J.M. Hoff da Silva, R. da Rocha, Eur. Phys. J. C 75, 266 (2015) 